\title{
Version définitive des directives et recomman- dations «Traitement et prise en charge des personnes âgées en situation de dépendance»
}

\section{A. Stuck ${ }^{a}$}

Die deutsche Fassung folgt.
La sous-commission «Traitement et prise en charge des personnes âgées en situation de dépendance» a été agréablement surprise par le nombre et la qualité des remarques et suggestions parvenues au secrétariat général. Elle a examiné avec soin tous les avis et en a tenu compte dans la mesure du possible:

\section{Commentaires rédactionnels}

Certains passages du texte ont semblé difficiles à comprendre et la répartition en directives et recommandations a quelquefois prêté à confusion.

\section{Réponse}

La rédaction du texte a été entièrement revue. Dans les directives et recommandations révisées, nous avons entre autres mis en évidence de façon uniforme les principales questions éthiques, afin de structurer le document et de rendre son utilisation plus aisée.

\section{Champ d'application des directives}

Plusieurs personnes auraient souhaité que les directives ne s'appliquent pas uniquement aux personnes âgées en situation de dépendance, mais également aux personnes jeunes en situation de dépendance. De même, certains ont déploré que les destinataires des directives et recommandations n'aient pas été définis plus clairement.

a Andreas Stuck est président de la sous-commission de l'ASSM chargée de l'élaboration de ces directives. Il est médecin-chef au Centre de réhabilitation en gériatrie de l'hôpital Ziegler à Berne et professeur honoraire en gériatrie à l'université de Berne.

1 Académie Suisse des Sciences Médicales. Traitement et prise en charge des personnes âgées en situation de dépendance. Bull Méd Suisses 2003;84(24):1292-302. (aves pourraient s'appliquer également à personnes jeunes, il existe des aspects qui relèvent d'autres exigences éthiques (par ex. les conséquences qu'une situation de dépendance peut avoir sur l'activité professionnelle ou des handicaps liés à une infirmité de naissance). En outre, lors de l'élaboration et de la consultation, nous avions fait appel à des experts issus du domaine de la gérontologie. C'est pourquoi, nous préférons limiter le champ d'application des directives aux personnes âgées. D’un autre côté, une définition claire des destinataires de ces directives et de leur champ d'application nous paraît importante, c'est pourquoi dans le texte révisé les destinataires (préambule) et le champ d'application (chapitre séparé) ont été définis de façon plus détaillée.

\section{Processus décisionnels en cas d'incapacité de discernement}

Plusieurs personnes ont estimé que le concept du représentant thérapeutique tel qu'il a été proposé est confus, voire problématique du point de vue juridique. A diverses reprises, il a été relevé que, pour la mise en pratique, les processus ne sont pas suffisamment détaillés (p.ex. en ce qui concerne la hiérarchie des compétences, la définition du «représentant thérapeutique»). En outre, l'importance de ces directives dans le contexte de la nouvelle loi sur la protection des adultes a été soulignée.

\section{Réponse}

Nous avons remanié les chapitres concernés et décrit avec plus de précision les processus décisionnels. A cet égard, nous avons notamment revu la définition du «représentant thérapeutique» ainsi que la marche à suivre lorsque des directives anticipées ont été rédigées. Nous avons en outre également clarifié la procédure en cas d'absence de représentant mandaté. Après avoir vérifié l'actualité des données juridiques, nous avons commenté dans une note en bas de page détaillée les réglementations légales en vigueur concernant les personnes âgées incapables de discernement n'ayant pas désigné de représentant thérapeutique.

\section{Assistance au suicide}

La thématique de l'assistance au suicide a fait couler beaucoup d'encre. A la lecture des directives, les dispositions légales en vigueur étaient quelque peu confuses pour certaines personnes. 
D’autres ont jugé inadéquats les conseils détaillés divulgués aux institutions quant au déroulement lui-même et ont exigé une séparation claire de la pratique du suicide assisté. Il est toutefois incontestable que les activités des organisations d'euthanasie auprès de personnes en situation de dépendance doivent être considérées d'un œil critique.

\section{Réponse}

Le texte sur l'assistance au suicide a été révisé. Le titre de ce chapitre a été modifié en «Attitude face à une demande de suicide»; ainsi la différence par rapport à la thématique de l'attitude suicidaire en tant qu'urgence médicale est clairement établie. Dans le texte révisé, le devoir de protection du personnel médical et de l'institution est décrit avec précision. Les questions fondamentales quant à l'assistance au suicide sont traitées dans le cadre des nouvelles directives «Prise en charge des personnes en fin de vie» de l'ASSM.

\section{Protection des données}

Des propositions concrètes concernant les domaines de la protection des données et de la documentation nous sont parvenues. Il a été souhaité que les directives ne se limitent pas aux aspects juridiques, mais démontrent également les dimensions éthiques de la protection des données.

\section{Réponse}

Dans les directives révisées, les aspects de la protection des données sont commentés avec plus de précision et selon les principes suivants: Il convient de s'assurer que les moyens mis en œuvre pour l'évaluation gériatrique multidimensionnelle sont proportionnés et relevants et d'informer les personnes âgées concernées de la collecte de ces données ainsi que de leur usage. Le dossier de soins et le dossier médical contiennent des données particulièrement confidentielles; celles-ci doivent être manipulées et conservées de telle façon que seules les personnes autorisées y aient accès.

\section{Mise en pratique des directives}

Nombreux sont ceux qui ont relevé la difficulté de mettre en pratique de telles directives sans la garantie d'un effectif du personnel suffisant. La formulation de conditions-cadre en matière de finances et de ressources humaines a été revendiquée à plusieurs reprises.

\section{Réponse}

Il va de soi que ces aspects sont déterminants voire essentiels quand il s'agit de la qualité du traitement et de la prise en charge des personnes âgées en situation de dépendance. D'un autre côté, des directives éthiques n'ont pas pour vocation de définir des normes obligatoires pour la pratique. Toutefois, il ressort de ces commentaires qu'il n'était pas toujours clair, s'il s'agit dans ces directives d'indications pour la pratique actuelle ou de perspectives idéales pour un futur plus ou moins éloigné. C'est pourquoi, nous avons expressément stipulé dans le préambule que les directives donnent des conseils à mettre en pratique (et non pas des perspectives idéales).

\section{Autres suggestions}

Dans plusieurs passages du texte, des précisions ou indications supplémentaires ont été souhaitées par différentes personnes ou organisations. Ainsi, par ex. quelles thérapies doivent être proposées à quel moment ou de quelle façon la documentation doit-elle être réglée exactement?

\section{Réponse}

Les directives énoncent des conditions-cadres conceptuelles bien fondées et renoncent à des descriptions détaillées susceptibles d'être controversées. Certains commentaires ont été ajoutés, par ex. en ce qui concerne la discussion ouverte des aspects spirituels ou l'évocation dans tout le texte des thérapeutes. 